\title{
On Heat Conduction with Phase Change: Accurate Explicit Numerical Method
}

\author{
N. Sadoun ${ }^{1,2}$, E. K. Si-Ahmed ${ }^{1,3 \dagger}$ and J. Legrand ${ }^{3}$ \\ ${ }^{1}$ Laboratoire de Mécanique des Fluides Théorique et Appliquée, Faculté de Physique, Université des Sciences et de \\ la Technologie Houari Boumediene-USTHB, B.P. 32, El-Alia, 16111, Alger, Algérie \\ ${ }^{2}$ Service Transferts, Interfaces et Procédés-TIPs, Faculté des Sciences Appliquées/Ecole Polytechnique, Université \\ Libre de Bruxelles-ULB, CP165/67, avenue F.D. Roosevelt 50, 1050 Bruxelles, Belgique \\ ${ }^{3}$ LUNAM Université, Université de Nantes, CNRS, GEPEA UMR-6144, CRTT, BP 40644602 Saint-Nazaire Cedex \\ - France
}

†Corresponding Author Email: esi-ahmed@usthb.dz.or El-khider.Si-ahmed@univ-nantes.fr

(Received July 16, 2010; accepted March 13, 2011)

\begin{abstract}
The one phase Stefan problem is discussed using the Goodman HBI method and an explicit numerical method including modified boundary immobilization scheme. The main advantage of the HBI method lie in the remarkable association of simplicity, flexibility and acceptable accuracy which an error less than $2.5 \%$ in predicting the moving front location for Stefan number less than unity which covers most usual isothermal phase change material. An accurate explicit numerical model to track the moving front in Stefan-like problems is provided. The scheme is obtained using the variable space step method based on variable domain. The method is developed using central difference approximations to replace spatial and temporal derivatives. Furthermore, iterative procedure, in numerical calculation, is avoided by introducing simple assumptions. The numerical results show that the accuracy of the method has been considerably improved without additional computational cost.
\end{abstract}

Keywords: Stefan, Moving boundary, Numerical method, Variable space step

\section{NOMENCLATURE}

$\begin{array}{ll}c & \text { specific heat } \\ e & \text { relative error } \\ h & \text { space step size } \\ k & \text { time step size } \\ M & \text { number of time stepping } \\ N & \text { number of space stepping } \\ s & \text { moving front dimensionless position } \\ \text { Ste } & \text { dimensionless latent heat }\end{array}$

\section{INTRODUCTION}

"One-phase Stefan problem" refers to heat conduction problem involving phase change in medium which is initially at its melting temperature and remains in thermal equilibrium during the phase change process. Heat transfer equations are then written in the formed phase only (where thickness changes with time according to the problem conditions). On the other hand, when heat transfer equations are to be considered in both solid and liquid phases the problem is referred as "two-phase Stefan problem" Carslaw and Jaeger (1959).

The solution of such problems is inherently difficult because of the nonlinear form of the thermal energy

\author{
$t \quad$ dimensionless time \\ $x \quad$ dimensionless coordinate \\ $i \quad$ space index \\ $j \quad$ time index during integration \\ $\Theta \quad$ dimensionless temperature \\ $\lambda$ freezing constant \\ $\zeta \quad$ shape function in temperature profile
}

balance equation at the solid/liquid interface. The latter's position being unknown, at first, must be followed as a part of the solution. Therefore, analytical solutions are, in general, difficult to obtain. As it is outlined in Sadoun et al. (2009), the 'Heat-Balance Integral' (HBI) is a technique that has received a considerable attention including aerodynamic heating by forced convection Sucec (1985); heat transfer with transpiration Zien (1976); prediction of the response of a positive temperature coefficient thermistor Kutluay et al (2006), Wood et al. (1995); conduction-controlled rewetting problems Sahu et al. (2006); boilover phenomenon occurring in fuel tanks Hristov ( 2007); nuclear reactor safety analysis El-Genk (1978); casting metals and spray forming Mitchell et al. (2008). On the overall, the method allows satisfactory results and, in some cases, could lead to analytical solutions. 
Furthermore, this technique shows remarkable flexibility to include cylindrical and spherical coordinates Poots (1962), Caldwell et al. (2000, 2005) with various boundary conditions and trials were carried on for two-dimensional Poots (1962) and threedimensional Riley et al.(1976) heat diffusion problems. The HBI method allows deriving the moving front velocity, heat fluxes or internal energy variations straightaway from the temperature profile. Such technique provides starting solutions for complex numerical schemes. The method appears, presently, to be a definite powerful approach to many nonlinear diffusion problems since it doesn't need any linearization of the required equations. It should be observed the nonlinearity can either be induced by the phase change at the interface, by the temperature dependency of the transport properties Goodman (1961), Vokov et al.(1970) or by the nonlinearity of the boundary conditions Goodman (1964). The main advantage is due to the transformation of the governing equation, from a partial derivative form to an ordinary differential equation where the assumed profile constitutes the central part of the original Goodman's technique (Goodman 1958; Sadoun et al. 2009). However, the method accuracy depends on the chosen profile.

Despite a large number of investigations (Goodman 1961; Poots 1962a; Vujanovic and Djukic 1972; Langford 1973; Fox 1975; Sadoun et al. 2006b; Sadoun and Si-Ahmed 1993,1995; Mosally et al. 2005) there is, unfortunately, no systematic procedure to choose the most appropriate profile; for that purpose works were oriented towards a decrease on the accuracy dependence of the method on an arbitrarily profile (Poots 1962, Hamil et al. 1963, Elmas 1970, vokov et al. 1970, Noble 1975, Bell 1978, Özisik 1985, Sadoun and Si-Ahmed 1993). Various approximate numerical methods are developed and have been the subject of many comprehensive books and papers Carslaw et al. (1959), sadoun (2006). These approximations can be conveniently handled into explicit or implicit methods according to the procedure used to localize the moving boundary.

In the implicit methods, the solution of the energy equation doesn't require the knowledge of the solid/liquid interface position which is implicitly deduced from the enthalpy or temperature distribution. These methods, called "one-phase methods" or "enthalpy methods" (Voller and Cross 1981, Elsen and Kutluay 2004), treat the solid/liquid domain as one phase for which the thermophysical properties are strongly dependant on temperature. The heat balance equation at the interface is implicitly bounded up in a new form of the energy equation available for the whole domain. On the other hand, in the explicit methods (Sadoun 2006; Savović and Caldwell 2003; Kutluay 2005), the governing equations are formulated for each phase (liquid and solid) and coupled at the interface by the temperature continuity and the energy balance. Then the moving front position constitutes a part of the problem solution and is to be tracked at each time step, making then the solution more complex because the nonlinear form of the energy balance equation at the interface. The methods are also referred to as 'two- phase methods" or "front-tracking methods".

In the present paper the one-phase Stefan problem with some refinement procedures, developed in the literature, are reported and compared. Furthermore a numerical explicit method which uses a technique based on front-tracking and referred to as variable space grid method (VSGM) is developed. Such method makes the numerical effort easier by subdividing the space into equal size intervals so that the moving boundary always remains at a grid point at each time step. Two Stefanlike problems are considered as physical models for application; the first concerns the classical one-phase Stefan problem with imposed temperature at the fixed boundary whereas the second deals with one-phase Stefan problem with periodically time dependent boundary condition. The paper's contribution aims to showing that the numerical scheme obtained by this method can lead to the solution for given initial time and space stepping. Furthermore, it considers the modified boundary immobilisation scheme used by Kutluay et al. (1997) and shows-up the lack of accuracy and proposes a way to make it as precise as the original scheme.

\section{Classical One-Phase Stefan Problem}

The classical mathematical model of one-phase Stefan problem refers to nonlinear diffusion equation. The nonlinearity is associated to the equation expressing the jump condition in terms of flux at the moving boundary. For the classical Stefan problem, in the case of the heat diffusion, the medium holds the half-plane $x \geq 0$ and the phase change is initiated at the boundary $x=0$ with a sudden temperature decrease (freezing) or increase (melting) and maintained fixed during all the process. The transport properties are considered constant.

The heat transport equation is then written in terms of dimensionless variables in the layer with appropriates initial and boundary conditions. The Stefan number, Ste $=(\rho c \Delta \Theta) /(\rho L)$, expressing the dimensionless latent heat is defined by the ratio of the heat needed to cool the solid to the latent heat required to transform the liquid to solid ( $c$ and $L$ refer to specific and latent heat respectively and $\Delta \Theta$ stands for temperature variation). The condition closing the mathematical model specifies that the moving front is at rest initially. The exact solution of this problem, available in literature (Carslaw and Jaeger 1958), can be easily derived by using the similarity variable to transform the governing equation from partial derivative to ordinary differential equation. Our interest is focused on the moving front position expressed by $s(\tau)=2 \lambda \sqrt{\tau}$ where $\lambda$ is the freezing constant given by the root of the transcendental equation $\lambda e^{\lambda} \operatorname{erf}(\lambda)=S t e / \sqrt{\pi}$ where erf is the error function. Finding an analytical and semianalytical expression for $\lambda$ constitutes the next section.

\subsection{Goodman HBI Solution}

The HBI is analogous to the momentum integral method; the formulation doesn't require any 
linearization of the considered equation. The analysis introduces the well known notion of penetration depth, beyond which there is no heat transferred. It is observed that, the latter, when no phase change is involved, is set instantaneously to infinity, according to Fourier's law. However, this is not the case in Stefan like-problem since the thermal depth penetration coincides with the phase change front.

The suitable approximating profile for the description of the temperature distribution in the layer is assumed to be quadratic. The technique is based on the approximation of the heat conduction equation by an overall energy balance in the domain considered. The result is referred to as the heat balance integral for the region of interest and expresses the macroscopic heat balance across this region. The technique satisfies the heat conduction equation only on the average. Moreover, derivatives of an arbitrarily profile may induce errors.

After substituting the profile in HBI equation which takes into account the Stefan condition, the moving boundary location is tracked through the following analytical expression:

$$
s(t)=\sqrt{2\left[\sqrt{(\text { Ste }+6)^{2}+12 S t e}-(S t e+6)\right]} \sqrt{t}
$$

One should note the computational simplicity introduced by the HBI procedure compared to the exact solution which, requires evaluations of both the freezing constant through numerical iterative sequences and tabulated functions (the error function). Moreover, as shown in Fig.1, the obtained

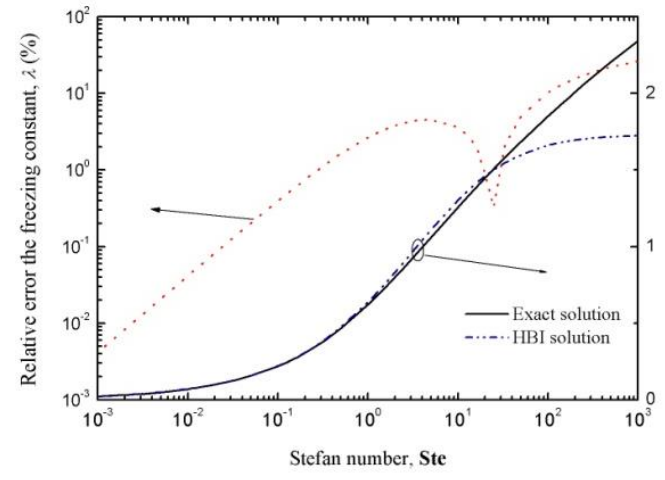

Fig. 1: Freezing constant, $\lambda$, as given by exact and HBI solutions and its associated relative error

$$
\left|\lambda_{\text {exa }}-\lambda_{\text {app }}\right| / \lambda_{\text {exa }}(\%)
$$

results from the above equation compared to the exact solution are satisfactory. The method predicts the moving front location with an error that does not exceed $2.5 \%$ for Stefan number less than unity which covers most usual isothermal phase change material. The relative error is defined as

$$
e_{s}=100 \frac{\left|\lambda_{\text {exa. }}-\lambda_{\text {app. }}\right|}{\lambda_{\text {exa. }}}
$$

These features motivated an appreciable number of investigations devoted to refinement procedures.

\subsection{Some analytical expressions for $\lambda$}

The various approaches developed, using Goodman HBI technique, to track the moving boundary in the case of the one-phase Stefan problem provided several approximate analytical solutions. Table 1 summarizes some analytical expressions of the freezing constant. On the overall, all expressions provide accurate localisation of the moving boundary for small Stefan numbers or at the beginning of the process (short times). This is due to the fact that the cumulated heat is negligible in both cases. Furthermore for small Ste the stored heat is small compared to latent heat while for short time the crust layer is very thin. In such cases the temperature profile can be well approximated by a simple linear form. Thus, these approaches provide an efficient device to compute starting solution for numerical schemes when required.

Table 1: Analytical freezing constant expressions obtained by analytical methods developed using heat balance integral technique

\begin{tabular}{|l|l|}
\hline \multicolumn{1}{|c|}{$\lambda^{2}$} & \multicolumn{1}{|c|}{ Authors } \\
\hline$\frac{1}{2}\left[\sqrt{(\text { Ste }+6)^{2}+12 \text { Ste }}-(\right.$ Ste +6$\left.)\right]$ & $($ Goodman 1961) \\
$3 \frac{1+2 \text { Ste }-\sqrt{1+2 \text { Ste }}}{5+2 \text { Ste }+\sqrt{1+2 \text { Ste }}}$ & \\
\hline $3 \frac{1+\text { Ste }-\sqrt{1+\text { Ste }}}{2+\text { Ste }+\sqrt{1+\text { Ste }}}$ & (Poots 1962a) \\
\hline$\frac{1+\text { Ste }-\sqrt{1+2 \text { Ste }}}{-1+2 \text { Ste }+\sqrt{1+2 \text { Ste }}}$ & (Wood 1995) \\
\hline$\frac{2 \text { Ste }}{4+\text { Ste }}$ & $\begin{array}{l}\text { (Mennig and } \\
\text { Ozişik 1985) }\end{array}$ \\
\hline$\frac{\text { Ste }}{2+\text { Ste }}$ & $\begin{array}{l}\text { (Hamill and } \\
\text { Bankoff 1963; } \\
\text { Elmas 1970) }\end{array}$ \\
\hline$\frac{\text { Ste }(2+\text { Ste }+2 \sqrt{1+\text { Ste }})}{8(1+\text { Ste })}$ & $\begin{array}{l}\text { (El-Genk and } \\
\text { Cronenberg } \\
1979)\end{array}$ \\
$\frac{3 \text { Ste }}{5+\text { Ste }+\sqrt{1+2 \text { Ste }}}$ & $\begin{array}{l}\text { (Sadoun and } \\
\text { Si-Ahmed 1995). }\end{array}$ \\
\hline$\frac{2(3+\text { Ste })}{\frac{1}{4}\left[\sqrt{(\text { Ste }+6)^{2}+24 \text { Ste }}-(\text { Ste }+6)\right.}$ & $\begin{array}{l}\text { (Sadoun et al. } \\
\text { 2006b) }\end{array}$ \\
\hline
\end{tabular}

Freezing constant obtained from exponential and Gaussian basic heat balance integral method and refined HBI with simple exponential distribution sadoun et al.(2006) are summarized in Table 2. It should be observed that the solution requires solving transcendental equations.

Simple exponential or quadratic profile leads to the same order of accuracy in the Goodman's basic HBI. Gaussian profile provides, as expected, more accurate solution. This can be understood since the latter is suggested by the exact solution. As matter of fact, the first term in the power series of the error function 
$\operatorname{erf}(\eta)$ is described by $\eta e^{\eta^{2}}$. However, in most cases the exact solution is unfortunately unknown beforehand.

Table 2: Semi-analytical freezing constant expressions obtained by analytical methods developed using heat balance integral technique

\begin{tabular}{|c|c|}
\hline $\begin{array}{l}\text { Equation } \\
\text { for } \lambda^{2}\end{array}$ & $\begin{array}{l}\text { Transcendental equation to be solved } \\
\text { Authors }\end{array}$ \\
\hline $\begin{array}{l}\frac{\text { Ste }}{2} \frac{\zeta e^{\zeta}}{e^{\zeta}-1} \\
\frac{\text { Ste }}{2}(1+2 \zeta)\end{array}$ & $\begin{array}{l}{[(1+\text { Ste }) \zeta-\text { Ste }] e^{2 \zeta}-(2 \zeta-\text { Ste }) e^{\zeta}+\zeta=0} \\
(1+2 \zeta)\left[2(1+\text { Ste }) \zeta e^{\zeta}+(1-\zeta) \text { Ste }\right]-2 \zeta=0 \\
\quad \text { (Mosally et al. } 2005)\end{array}$ \\
\hline$\frac{\text { Ste }}{2} \zeta$ & $\begin{array}{c}\text { Ste } \zeta^{2}+(2 \text { Ste }+6) \zeta+\sqrt{\frac{72(1-\zeta)}{\text { Ste }}}-12=0 \\
\zeta \in] 0,1[ \\
(\text { Wood 1985). }\end{array}$ \\
\hline$\frac{\text { Ste }}{2} \frac{\zeta e^{\zeta}}{e^{\zeta}-1}$ & $\begin{array}{l}\zeta\left(e^{\zeta}-1\right)\left(\zeta e^{\zeta}-e^{\zeta}+1\right)+\operatorname{Ste}\left[\zeta(\zeta-2) e^{\zeta}+2\left(e^{\zeta}-1\right)\right] e^{\zeta}=0 \\
(\text { Sadoun et al. 2006a) }\end{array}$ \\
\hline
\end{tabular}

Figure 2 shows the relative error for the freezing constant using various correlations.

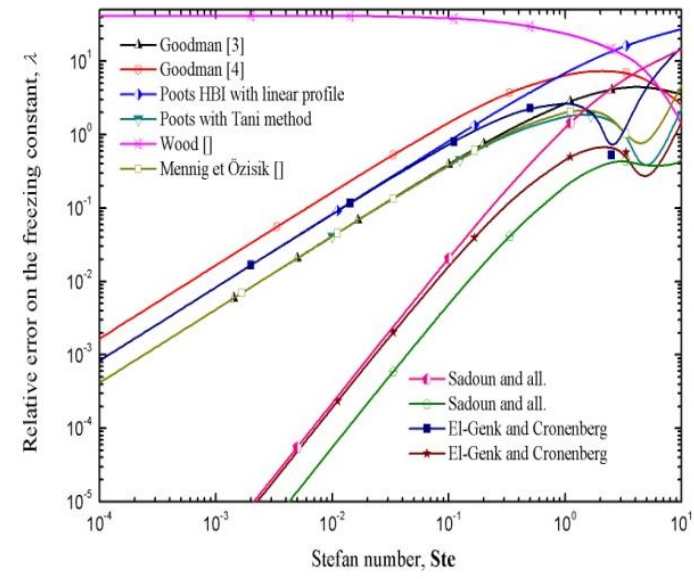

Fig. 2: Relative error $\left|\lambda_{\text {exa }}-\lambda_{\text {app }}\right| / \lambda_{\text {exa }}(\%)$ on the freezing constant, $\lambda$, as given by different approaches constructed using Goodman's HBI technique

\section{Modified VARIable SPaCe SteP Method For One- Phase Stefan Problem}

\subsection{Problem Statement}

Consider the freezing of semi-infinite liquid, occupying the region $x \geq 0$ and is initially at its melting temperature $\Theta(x, t)=0$. Then solidification is initiated instantaneously since the fixed boundary $x=0$ is raised at $t=0$ to a temperature above the melting temperature and subsequently maintained fixed or timedependent. If we note by $s$ the position of the freezing front at time $t$ and assume than the process is controlled by the heat conduction, then the temperature distribution $\Theta(x, t)$ is expressed as: $\frac{\partial \Theta(x, t)}{\partial t}=\frac{\partial^{2} \Theta(x, t)}{\partial x^{2}} ; \quad 0<x<s(t)$

with the related Stefan condition (case of Ste $=1$ )

$$
\frac{d s(t)}{d t}=-\frac{\partial \Theta(x, t)}{\partial x} ; \quad x=s(t) ; t>0
$$

\subsubsection{Problem1:Classical one-phase Stefan problem}

The classical one-phase Stefan problem is considered here with fixed boundary raised up, from $t=0$, above the melting temperature. Mathematically that is expressed by the following dimensionless equation.

$\Theta(0, t)=1 ; x=0 ; t \geq 0$

The exact analytical solution can be deduced from the generalized Neumann solution (Carslaw and Jaeger 1959) as follows:

$\Theta(x, t)=1-\frac{\operatorname{erf}\{x /(2 \lambda \sqrt{t})\}}{\operatorname{erf}(\lambda)}$

Where the moving boundary position $s(t)$ is obtained from the following expression

$s(t)=2 \lambda \sqrt{t}$

with a freezing constant $\lambda$ being the root of the transcendental equation $\lambda e^{\lambda^{2}} \operatorname{erf}(\lambda) \sqrt{\pi}=1$

\subsubsection{Problem2: One-phase Stefan problem with time- dependent boundary condition}

The second problem considers one-phase Stefan problem with periodically time-dependent boundary temperature:

$$
\Theta(0, t)=1-0.5 \sin (0.5 \pi t) ; x=0 ; t \geq 0
$$

Taking into account that no exact solution is available in the literature for such case, the numerical results will be compared to the analytical solution developed using the refined heat balance integral method with a linear approximant Sadoun et al. (2006); that is:

$$
\Theta(x, t)=\Theta(0, t)\left(1-\frac{x}{s(t)}\right)
$$

Where $s(t)$ is given by:

$$
s(t)=\sqrt{\frac{6}{3+U(0, t)} \int_{0}^{t} \Theta(0, t) d t}
$$

For both problems, the method leads to analytical solutions. In the case of one-phase classical Stefan problem (problem 1), the numerical results are in good agreement with the exact solution particularly at the beginning of the phase change process (for thin layer) (Sadoun et al. 2006b).

\section{2 Numerical Solution}

Following Murray and Landis (1959), the mathematical model is written over by applying partial derivative 
with respect to time, tracking then a given line instead of a constant $x$. The heat conduction equation becomes

$$
\left.\frac{\partial \Theta}{\partial t}\right|_{x}=\left.\frac{d s}{d t} \frac{x_{i}}{s} \frac{\partial \Theta}{\partial x}\right|_{t}+\left.\frac{\partial^{2} \Theta}{\partial x^{2}}\right|_{t} ; 0<x<s(t)
$$

Related initial and boundary conditions remains unchanged. It should be noted that suffices $x$ and $t$ indicate constant values.

Various numerical schemes can be deduced from the above governing equations (Crank 1957). The variable step grid method is considered hereafter. This is achieved by locating, through a mathematical model, the liquid/solid interface at given moving grid point. The transformed heat diffusion equation (Eq.9) will be solved by partial discretization using central difference approximations to replace spatial and temporal derivatives. Also, a three-term central difference formula is used for numerical evaluation of the temperature gradient in the energy balance at the moving boundary.

In this method, the time interval $k$ is kept constant and the space domain $0<x<s(t)$ is divided into $N$ intervals of width $h$ with $N h=s(t)$. Thus, the moving boundary is always located at the last grid point by increasing the space width step with the growth of the solidified layer. If we put $h^{j}$ the size at the time $j k$ $j=1,2, \ldots$, then any point $\left(x_{i}, t_{j}\right)$ in $x-t$ domain is given by $\left(i h^{j}, j k\right)$ and $\Theta_{i}^{j}(i=0,1, \ldots, N)$ denotes the finite difference approximation of its temperature.

After a few arrangements according to the above mentioned discretization procedure, Eqs. (1-2) in terms of finite differences leads respectively to:

$$
\begin{aligned}
& \Theta_{i}^{j+1}=U_{i}^{j}+\frac{i}{2} \frac{k}{h^{j+1 / 2}}\left(\frac{d h}{d t}\right)^{j+1 / 2}\left(\Theta_{i+1}^{j+1 / 2}-\Theta_{i-1}^{j+1 / 2}\right) \\
& +\frac{k}{\left(h^{j+1 / 2}\right)^{2}}\left(\Theta_{i+1}^{j+1 / 2}-2 \Theta_{i}^{j+1 / 2}+\Theta_{i-1}^{j+1 / 2}\right) \\
& \left(\frac{d h}{d t}\right)^{j+1 / 2}=\frac{4 \Theta_{N-1}^{j+1 / 2}-\Theta_{N-2}^{j+1 / 2}}{N h^{j+1 / 2}}
\end{aligned}
$$

It should be noted that eq. (11) takes into account the moving boundary condition $\left(\Theta_{N}(t)=0\right.$ for $\left.t \geq 0\right)$ and allows locating the moving boundary at the next time step according to following approximation

$h^{j+1}=h^{j}+\frac{4 \Theta_{N-1}^{j+1 / 2}-\Theta_{N-2}^{j+1 / 2}}{N h^{j+1 / 2}}$

To avoid numerical iterations, we assume that the variation of the nodal temperature, $\Theta_{i}$, with time and along a half time step is negligible. The temperatures $\Theta_{i-1}^{j+1 / 2}, \Theta_{i}^{j+1 / 2}$ and $\Theta_{i+1}^{j+1 / 2}$ are then replaced by $\Theta_{i-1}^{j}$, $\Theta_{i}^{j}$ and $\Theta_{i+1}^{j}$ respectively. Furthermore, the step size $h^{j+1 / 2}$ is approached by the average value of $h^{j}$ and $h^{j+1}$ as follows

$h^{j+1 / 2}=\left(h^{j+1}+h^{j}\right) / 2$
Rearranging Eq.(11) to lead to the new front position location:

$h^{j+1}=\sqrt{\left(h^{j}\right)^{2}+\frac{2}{N}\left(4 \Theta_{N-1}^{j}-\Theta_{N-2}^{j}\right)}$

and Eqs. (10-11) could be written as:

$\left(\frac{d h}{d t}\right)^{j+1 / 2}=\frac{4 \Theta_{N-1}^{j}-\Theta_{N-2}^{j}}{N h^{j+1 / 2}}$

$\Theta_{i}^{j+1}=\Theta_{i}^{j}+\frac{i}{2} \frac{k}{h^{j+1 / 2}}\left(\frac{d h}{d t}\right)^{j+1 / 2}\left(\Theta_{i+1}^{j}-\Theta_{i-1}^{j}\right)$

$+\frac{k}{\left(h^{j+1 / 2}\right)^{2}}\left(\Theta_{i+1}^{j}-2 \Theta_{i}^{j}+\Theta_{i-1}^{j}\right)$

The system of equations is closed with the given condition $\Theta(0, t)$ at the fixed boundary $x=0$.

The resulting explicit numerical scheme is somewhat similar to those developed by Kutluay et al. [1997, 2004, 2005]; Elsen et al. , 2004, Savović et al 2003; except that the present one uses both freezing front velocity and location at the time $t^{j+1 / 2}$ instead of their values at time $t^{j}=j k$.

From known values of $\Theta_{i}^{j} \quad(i=0, \ldots, N)$ and $s^{j}$ at time $t^{j}=j k$, the solution procedure involves:

i)Calculation of space step size $h^{j}=s^{j} / N$ and the grid point locations $x_{i}^{j}=i h^{j}$.

ii)Determination of the step size $h^{j+1}$ using Eq.(14) and then interpolation of $h^{j+1 / 2}$ from Eq.(13).

iii)Calculation of the freezing front velocity at time $t^{j+1 / 2}=(j+1 / 2) k$ using Eq. (15).

iv)Solution of Eq.(16) for the grid points $i=1, \ldots, N-1$.

The steps (i)-(iv) are repeated for the next step after setting the performed values as starting solution

\section{RESULTS AND DISCUSSION}

\subsection{Starting solutions}

To start numerical calculations for both problems, and to circumvent the singularity at time $t=0$, i.e. $s(t)=0$, we used the temperature distribution and the corresponding position of the freezing front at $t_{1}=0.1$ which is used in Kutluay et al. $(1997,2005)$ as the initiated time. The corresponding nodal temperatures, $\Theta_{i}^{j}=\Theta\left(i h, t_{1}\right)$, and moving boundary position, $s^{1}=s\left(t_{1}\right)$, are determined from the exact Eqs. $(4,5)$ and analytical Eqs. $(7,8)$ solutions for the problems 1 and 2 respectively. One can note that $M=\left(t-t_{1}\right) / k$ time steps are to be used to obtain the numerical solution at a given time $t$. 


\subsection{Numerical Results}

The weighted 1-norme $\|e\|_{1}$ and the relative error on the moving front location, $e_{x}$, defined below are considered to test the accuracy of the obtained numerical scheme and to compare against available results in the Refs. $[8,9,10]$

$$
\begin{aligned}
& \|e\|_{1}=\frac{1}{N-1} \sum_{1}^{N-1}\left|1-\frac{\Theta_{i}^{j}}{\Theta\left(x_{i}, t^{j}\right)}\right|, e=\left[e_{1} e_{2} \ldots e_{N-1}\right]^{T}, \\
& \text { and } e_{s}=10^{2}\left|1-\frac{s^{j}}{s\left(t^{j}\right)}\right| \text {;Where } s\left(t_{j}\right) \text { and } \quad \Theta\left(x, t^{j}\right)
\end{aligned}
$$

are the exact values.

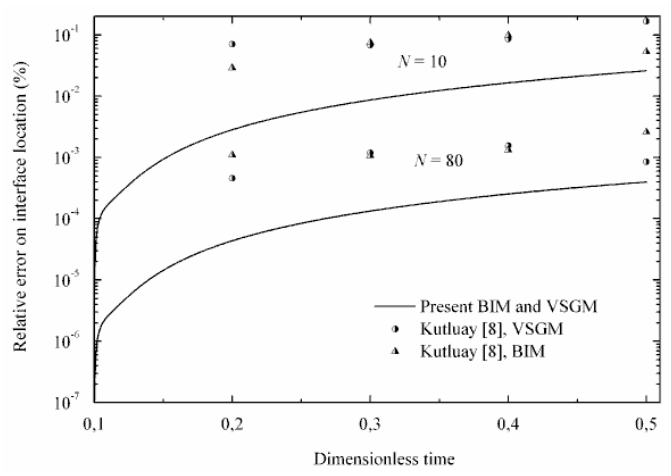

Fig. 3: Evolution of the relative error on the moving boundary position for two space stepping in the case of the problem 1

Figures 3 and 4 show the variation of the relative error on the moving boundary position, $s$, with time, $t$. Different numbers of space subdivisions $N$ are considered. It can be seen that for a given space subdivision, the numerical predictions of the interface location depart from the exact positions as time increases but still sufficiently accurate. On the other hand, Fig. 3 compares accuracies of the present method with those given by modified VSGM and BIM developed by Kutluay and al. $(1997,2005)$

The plots demonstrate clearly that the present scheme improves considerably the accuracy of the numerical solution. The figures exhibit the expected convergence of the scheme with the refinement of the mesh size. One can note, from Fig. 4, that increasing $n$ times the number of space intervals refines the accuracy about $n^{2}$-fold ( $n$ corresponds to the ratio of the two corresponding time steps).

Figure 5 illustrates the dependence of the weighted 1norme error $\|e\|_{1}$ on the temperature defined below on the number of space subdivisions, and computed at time $t=0.5$. One can note the rapid decrease of the error with the increase of the number of subdivisions. The vertical dashed line on the figure indicates the number minimum of subdivisions allowed by the numerical scheme, that is $N=3$.

The numerical results given by the present scheme are displayed in Table 3, with a comparison of three other numerical solutions available in the literature at final times $0.2,0.3,0.4$ and 0.5 . From data, it's observed that the results exhibit the convergence as the space stepping is reduced. It's clearly seen that the present scheme generates more accurate solutions than schemes developed using MVSGM, BIM and IMM.

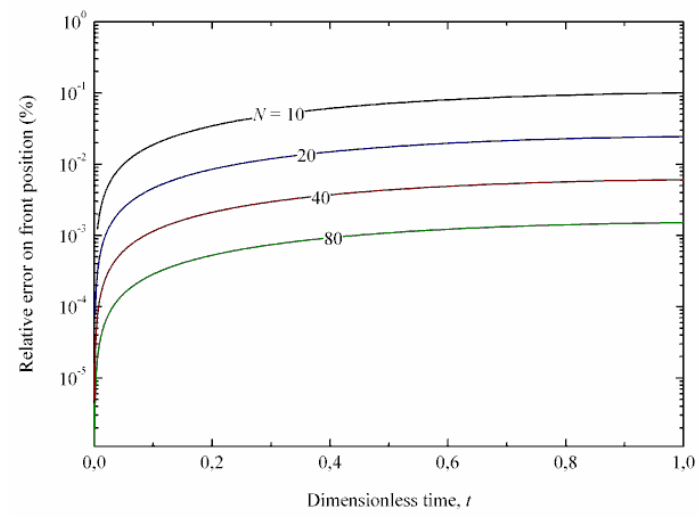

Fig. 4: Evolution of the relative error on the moving boundary position for different space stepping in the case of the problem 1

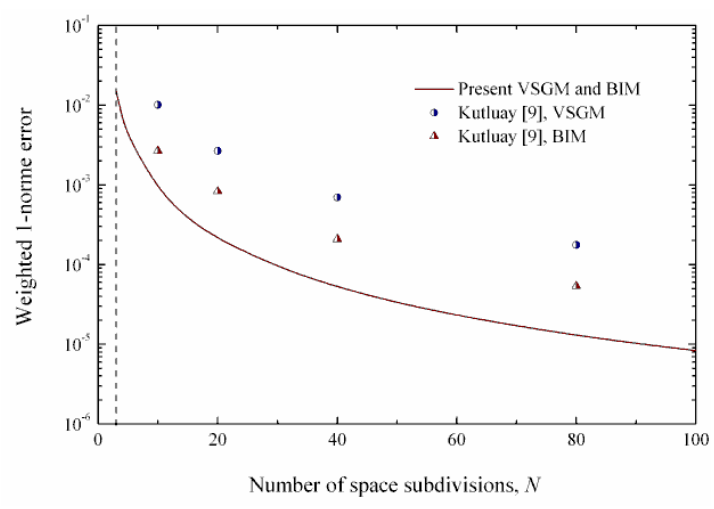

Fig. 5: Weighted 1-norme error $\|e\|_{1}$ on the temperature versus the number of space subdivisions for the problem 1 at time $t=0.5$.

Finally, the scheme is considered in the case of the second problem. Figure 6 shows the time evolution of the moving front boundary as given by the numerical and analytical solution Sadoun et al. (2006). It's clearly seen that they are very close to each other.

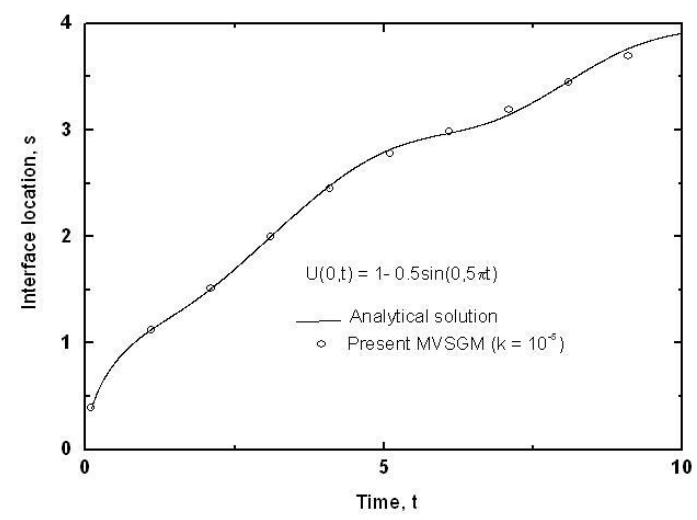

Fig. 6: Numerical and analytical moving front position pre-dictions for the problem 2 


\section{CONCLUDING REMARKS}

The computational simplicity introduced by the HBI procedure compared to the exact solution was highlighted. The results showed that the method predicts the moving front location with an error that does not exceed $2.5 \%$ for Stefan number less than unity which covers most usual isothermal phase change material. Furthermore, a new explicit numerical scheme is developed using the variable space method.
Despite the use of central difference approximation to replace both spatial and temporal partial derivatives, the numerical scheme is expressed in explicit form avoiding then the iterative procedure to achieve numerical calculation. The numerical results obtained in the case of two Stefan like problems demonstrate the good accuracy of the present scheme.

Table 3 Comparison of the relative error of predicted location of the moving boundary with results from the present method, the modified variable step method (MVSGM) (Kutluay 2005), boundary immobilization method (BIM) (Kutluay et al. 2005)and isotherm migration method (IMM) (Kutluay and Elsen 2004) at various times and mesh sizes for the problem 1

\begin{tabular}{|c|r|c|c|c|c|}
\hline \multirow{2}{*}{$t$} & \multirow{2}{*}{ method } & \multicolumn{4}{|c|}{ Relative error, $e_{s}(\%)$} \\
\cline { 3 - 6 } & & $N=10$ & $N=20$ & $N=40$ & $N=80$ \\
\hline \multirow{4}{*}{0.2} & Present & 0.002831 & 0.000400 & 0.000096 & 0.000024 \\
& VSGM & 0.028870 & 0.007240 & 0.001810 & 0.000455 \\
& BIM & 0.070370 & 0.017610 & 0.004405 & 0.001100 \\
& IMM & 0.012555 & 0.003230 & 0.000820 & 0.000205 \\
\hline \multirow{4}{*}{0.3} & Present & 0.002035 & 0.000401 & 0.000087 & 0.000020 \\
& VSGM & 0.075487 & 0.018940 & 0.004740 & 0.001187 \\
& BIM & 0.068353 & 0.017093 & 0.004273 & 0.001067 \\
& IMM & 0.019093 & 0.005120 & 0.001320 & 0.000337 \\
\hline \multirow{4}{*}{0.4} & Present & 0.005464 & 0.001101 & 0.000243 & 0.000047 \\
& VSGM & 0.098720 & 0.024820 & 0.006218 & 0.001555 \\
& BIM & 0.085112 & 0.021268 & 0.005312 & 0.001328 \\
& IMM & 0.024728 & 0.006997 & 0.001848 & 0.000472 \\
\hline \multirow{3}{*}{0.5} & Present & 0.012830 & 0.002670 & 0.000602 & 0.000143 \\
& VSGM & 0.052922 & 0.013452 & 0.003386 & 0.000850 \\
& BIM & 0.166242 & 0.041576 & 0.010386 & 0.002596 \\
& IMM & 0.031792 & 0.009490 & 0.002558 & 0.000662 \\
\hline
\end{tabular}

\section{REFERENCES}

Bell, G. E., (1978). A refinement of the heat balance integral method applied to melting problem. Int. J. Heat Mass Transfer, 21, 1357-1362

Caldwell, J. and Y.Y. Kwan, (2005). Starting solutions for the boundary immobilisation method. Commun. Numer. Meth. Eng, 21, 289-295.

Caldwell, J. and C.C. Chan (2000). Spherical solidification by enthalpy method and the heat balance integral method. Appl. Math. Modelling, $24,45-53$

Carslaw, H.S. and J.C. Jaeger (1959). Conduction Of Heat In Solids. Second Edn. Oxford Clarendon Press, Oxford.

Crank, J. (1957). Two methods for the numerical solution of moving-boundary problems in diffusion and heat flow. Quart. J. Mech. Math., 10, 220-231.

Elmas, M. (1970). On the solidification of the warm liquid flowing over a cold wall. Int. J. Heat Mass Transfer, 13, 1060-1062

Elsen, A. and S. Kutluay (2004). A numerical solution of the Stefan problem with a Neumann-type boundary condition by enthalpy method. Appl. Math. Comput., 148, 321-329.
El-Genk, M. S. and Cronenberg, A. W. (1979). Some Improvements to the solution of Stefan likeproblems, Int. J. Heat Mass Transfer, 22, 167-17.

El-Genk, M.S. (1978). Improvements to the Solution of Stefan-like Freezing and Melting Problems, with Application to LMFBR Safety Analysis, Approximate Analysis of Heat Transfer in Transpired Boundary Layers with Effects of Prandtl Number. Ph.D. Dissertation, University of New Mexico, Albuquerque NM, USA,

Fox, L. (1975). What are the best numerical methods? Moving Boundary Problems In Heat Flow And Diffusion. Ed. Ockendon J. R., Hodgkins W. R., Clarendon Press, Oxford, UK, 210-241.

Goodman, T.R. (1964). Application of integral methods to transient nonlinear heat transfer. Advances In Heat transfer, Irvine T.F. Jr. \& Hartnett J P, eds., New York: Academic Press, Vol. I, 51-122.

Goodman, T.R. (1961). The heat balance integral further considerations and refinements. Trans. ASME J. of Heat Transfer, 83, 83-88.

Hamill, T. D. and S.G. Bankoff (1963). Maximum and minimum bounds of freezing-melting rates with time dependent boundary conditions. A.I.Ch.E. Journal, 9, 741-744. 
Hristov, J. (2007). An inverse Stefan problem relevant to boilover: Heat balance integral solutions and analysis. Thermal Science, 11, 141-160.

Kutluay, S., A.R Bahadir and A. Özdeş (1997)., The numerical solution of one-classical Stefan problem. J. Comput. Appl. Math., 81, 135-144.

Kutluay, S. and A. Elsen (2004). An isotherm migration formulation for one-phase Stefan problem with time dependent Neumann condition. Appl. Math. Comput., 150, 59-67.

Kutluay, S. (2005). Numerical schemes for onedimensional Stefan-like problems with a forcing term. Appl. Math. Comput., 168, 1159-1168

Kutluay, S., A.S. Wood and A. Esen (2006). A heat balance integral solution the termistor problem with a modified electrical conductivity. Appl. Math. Modelling, 30, 386-394

Langford D., The heat balance integral method, Int. J. Heat Mass Transfer, 16, 2424-2428 (1973).

Mennig, J. and M.N. Özişik (1985). Coupled integral approach for solving melting and solidification, Int. J. Heat Mass Transfer, 28, 1481-1485

Mitchell S.L. and T.G. Myers (2008). Approximate methods for one-dimensional solidification from an incoming fluid. Appl. Math. Comput., doi : 10.1016 / j.amc. 2008.02.031.

Mosally, F., A.S. Wood and A. Al-Fhaid (2005). An exponential heat balance integral method. Appl. Math. Comput., 29, 903-9012

Murray, W.D. and F. Landis (1959). Numerical and machine solutions of the transient heat conduction problems involving melting or freezing. J. Heat transfer, 81, 106-112.

Noble, B. (1975). Heat balance methods in melting problems. Moving Boundary Problems in Heat Flow and Diffusion, Ed. Ockendon J. R., Hodgkins W. R. , Clarendon Press, Oxford, UK, 208-209.

Poots, G. (1962a). On the application of integralmethods to the solution of problems involving the solidification of liquids initially at fusion temperature. Int. J. Heat Mass Transfer, 5, 525531.

Poots, G. (1962b). An approximate treatment of a heat conduction problem involving a two-dimensional front. Int. J. Heat Mass Transfer, 5, 339-348

Riley, D. S. and P.W. Duck (1976). Application of the heat-balance integral method to the freezing of a cuboid. Int. J. Heat Mass Transfer, 19, 294-296.

Sadoun, N. and E.K. Si-Ahmed (1993). On the double integral method for solving Stefan like-problems. Proceedings, First International Thermal and Energy Congress, Marrakesh, Morocco, Vol. 1, 87-91.
Sadoun, N. (2006). Contribution à l'Etude du Problème de Solidification - Approche Semi Analytique. Magister Thesis, Faculté de Physique, University of USTHB.

Sadoun, N., E.K. Si-Ahmed and J. Legrand. (2006a). A refined exponential heat balance integral method for one-phase Stefan problem. Proceedings, The Second International Conference on Thermal Engineering : Theory and Applications, Ed. Chacha and al., Al-Ain, UAE, 528-532

Sadoun, N., E.K. Si-Ahmed and P. Colinet (2006b). On the refined integral method for one-phase Stefan with time-dependent boundary conditions. Appl. Math. Modelling. 30, 531-544

Sadoun, N., E.K. Si-Ahmed, P. Colinet and J. Legrand (2009). On the Goodman heat-balance integral method for Stefan like-problems: Further considerations and refinements. Thermal Science, $13,81-96$

Sahu, S.K., P.K. Das and S. Bhattacharyya (2006). A comprehensive analysis of conduction-controlled rewetting by the heat balance integral method. Int. J. Heat Mass Transfer., 49, 4978-4986.

Savović, S. and J. Caldwell (2003). Finite difference solution of one-dimensional Stefan problem with periodic boundary conditions. Int. J. Heat. Transfer, 46, 2911-2916.

Sucec, J. (1979). Extension of modified integral method to boundary conditions of prescribed surface heat flux. Int. J. Heat Mass Transfer, 22, 771-774.

Verma, A.K., S. Chandra and B.K. Dhindaw (2004). An alternative fixed grid method for solution of the classical one-phase Stefan problem. Appl. Math. Comput., 158, 573-584

Vokov, V.N. and V.K. Li-Orlov (1970). A refinement of the integral method in solving the heat conduction equation. Heat Transfer Sov. Res., 2, 41-47.

Voller V. and M. Cross (1981). Accurate solutions of moving boundary problems using the enthalpie method. Int. J. Heat Mass Transfer, 24, 545-556

Vujanovic, B. and Dj. Djukic (1972). On one variational principle of Hamilton's type for nonlinear heat transfer problem. Int. J. Heat Mass Transfer, 15, 1111-1123.

Wood, A.S. and S. Kutluay (1995). A heat balance integral model for thermistor, Int. J. Heat Mass Transfer, 38, 1831-1840.

Zien, T.F. (1976). Approximate analysis of heat transfer in transpired boundary layers with effects of Prandtl number. Int. J. Heat Mass Transfer, 19, 513-521. 